\title{
Influence of serum vitamin D status on severe sarcopenia among female patients with rheumatoid arthritis: the KURAMA database
}

Hiroto Minamino

Kyoto University

Masao Katsushima

Kyoto University

Mie Torii ( $\square$ torii.mie.4x@kyoto-u.ac.jp )

Kyoto University

Wataru Yamamoto

Kurashiki Sweet Hospital

Yoshihito Fujita

Kyoto University

Kaori Ikeda

Kyoto University

Emi Okamura

Kyoto University

Kosaku Murakami

Kyoto University

Ryu Watanabe

Kyoto University

Koichi Murata

Kyoto University

Hiromu Ito

Kyoto University

Masao Tanaka

Kyoto University

Hidenori Arai

National Center for Geriatrics and Gerontology

Shuichi Matsuda

Kyoto University

Akio Morinobu

Kyoto University

Nobuya Inagaki 
Kyoto University

Motomu Hashimoto

Osaka City University

\section{Research Article}

Keywords: Vitamin D, 25(OH)D, Sarcopenia, Severe Sarcopenia, Rheumatoid Arthritis, Gait speed, Handgrip strength, Skeletal muscle mass index

Posted Date: July 13th, 2021

DOl: https://doi.org/10.21203/rs.3.rs-697981/v1

License: (a) This work is licensed under a Creative Commons Attribution 4.0 International License. Read Full License 


\section{Abstract}

Sarcopenia is an age-related disease with an increased risk of mortality. It is emerging that low serum vitamin $\mathrm{D}(25(\mathrm{OH}) \mathrm{D})$ affects the sarcopenic state in general, but in rheumatoid arthritis (RA), these associations are not understood although the prevalence of vitamin D insufficiency is high in RA. We conducted a cross-sectional study of older female outpatients from our cohort (KURAMA) database. We measured skeletal muscle mass, handgrip strength, and gait-speed to diagnose severe sarcopenia. The serum 25(OH)D concentration was measured using electrochemiluminescence immunoassay. A total of 156 Patients (sarcopenia:44.9\%, severe sarcopenia: $29.5 \%$, and without sarcopenia: $25.6 \%$ ) were enrolled. Classification of vitamin D status at a cutoff point of median $25(\mathrm{OH}) \mathrm{D}$ concentration revealed that low $25(\mathrm{OH}) \mathrm{D}$ status was associated with a high prevalence of severe sarcopenia and with low measured values of muscle mass, handgrip, and gait-speed. Furthermore, multiple regression analysis identified that low 25(OH)D status was associated with a high prevalence of severe sarcopenia (OR $6.00 ; 95 \% \mathrm{Cl}$ 1.99-18.08). In components of sarcopenia, both low physical performance and muscle mass were associated with low $25(\mathrm{OH})$ status.

In conclusion, vitamin D levels are associated with severe sarcopenia and its components, and modification of vitamin $D$ status including vitamin $D$ supplementation may play a role in improving sarcopenia in RA.

\section{Introduction}

Sarcopenia is an age-related disease characterized by poor physical performance and reduced muscle mass and strength, and one of the most important and challenging problems in an aging society. Sarcopenia has multifactorial etiology including malnutrition, aging, infrequent exercise and diseases such as cancer, diabetes mellitus, COPD and autoimmune disease ${ }^{1}$; it is closely related to increased risk of mortality ${ }^{2,3}$. Although some therapeutic interventions are established such as encouragement of protein intake and physical exercise in the general population 4,5 , there still remain unknown aspects of sarcopenia in patients with debilitating diseases.

RA is a inflammatory autoimmune disease characterized by increased morbidity and mortality due to joint destruction and extra-articular manifestations. RA patients exhibit far more sarcopenia than the general population ${ }^{6}$ and loss of physical performance such as walking and grip strength is a well-known risk factor of mortality ${ }^{7}$. Severe sarcopenia, which requires more intensive interventions to achieve improvements in physical performance ${ }^{8,9}$, is highly prevalent in RA patients compared to the general populations ${ }^{10,11}$. There have been conflicting results as to whether Disease Modifying Anti-Rheumatic Drugs (DMARDs) and biological agents improve the sarcopenic state in RA patients ${ }^{10,12}$; there is also little evidence on co-adjuvant therapies such as nutritional interventions in these patients.

Recently, vitamin D supplementation is receiving much attention as a potential therapeutic intervention for preventing sarcopenia in the general population in addition to protein supplementation and exercise. 
Vitamin D may well ameliorate the sarcopenic state via its role in muscle cell regulation, antiinflammatory pathways and/or immunomodulatory responses ${ }^{13}$, as serum vitamin $D$ levels are associated with muscle strength and physical performance ${ }^{14,15}$. Indeed, meta-analyses and RCTs have shown that vitamin D supplementation improves limb strength in the community-dwelling elderly 16,17 . Interestingly, over $70 \%$ of RA patients exhibit vitamin D insufficiency ${ }^{18}$; these findings suggest the importance of vitamin D on sarcopenia in RA populations as in general populations. However, it remains unclear whether serum vitamin D levels affect the sarcopenic state in RA patients.

To elucidate the clinical association between vitamin D deficiency and sarcopenia in elderly patients with RA, we performed a cross-sectional study using the cohort study database (KURAMA cohort).

\section{Methods}

\section{Study design and participants}

We conducted a cross-sectional study in female RA outpatients from the Kyoto University Rheumatoid Arthritis Management Alliance (KURAMA) cohort database, which has been described in detail elsewhere 19,20. We recruited older RA participants who visited the Kyoto University Hospital from May 2014 to December 2014 and who were over 60 years of age. All participants fulfilled the diagnostic criteria of the ACR/EULAR RA classification ${ }^{21}$. Of the total of 248 participants, 10 were excluded because of incomplete data set (lack of preserved serum or parameters of sarcopenia-related factors) and 82 were excluded because of the use of vitamin $D$ supplementation. The remaining 156 participants were subjected to the analysis

\section{Ethics}

The study protocol and procedures were approved by the Medical Ethics Committee of Kyoto University Graduate School and Faculty of Medicine (Approval number: R0357) and complied with the principles of the Declaration of Helsinki. We obtained ritten informed consent was obtained from all patients of this study, which included the use of human blood samples and data.

\section{Diagnosis of sarcopenia and estimation of the related parameters}

We measured muscle mass, muscle strength and physical performance to assess sarcopenia status in the study population, as previously described ${ }^{10,22}$. Skeletal muscle mass was measured by bioelectrical impedance analysis (Inbody 720: Biospace Co., Ltd., Seoul, Korea). Skeletal muscle index (SMI) was computed from the appendicular skeletal muscle mass in kilograms divided by the square of height in meters $\left(\mathrm{kg} / \mathrm{m}^{2}\right)$. Muscle strength was measured by handgrip strength using JAMAR digital hand dynamometer (Patterson Medical, Bolingbrook, IL). Physical performance was also measured by the 6meter walking speed using a portable gait rhythmogram (MG-M1110: LSI Medience Co., Tokyo, Japan) 
Regarding muscle strength and gait speed, the mean value of duplicate measurements was used for analysis.

Diagnosis of sarcopenia and severe sarcopenia were based on the criteria of the Asian Working Group for Sarcopenia (AWGS) $2019^{23}$. Briefly, sarcopenia was defined as low muscle mass with low muscle strength or with low physical performance; severe sarcopenia was defined as low muscle mass with both low muscle strength and low physical performance. The cutoff values of low muscle mass, low muscle strength and low physical performance were $\mathrm{SMI}<5.7 \mathrm{~kg} / \mathrm{m}^{2}$, handgrip strength $<18 \mathrm{~kg}$, and gait speed $<$ $1.0 \mathrm{~m} / \mathrm{s}$, respectively.

\section{Evaluation of vitamin D level and the clinical parameters}

Serum 25-hydroxyvitamin D (25(OH)D), an established biomarker reflecting vitamin D status, was measured using electrochemiluminescence immunoassay (LSI Medience Co., Tokyo, Japan). MNA-SF (Mini Nutritional Assessment Short-Form) was collected to assess nutritional state ${ }^{24}$. RA disease activity and physical dysfunction were assessed by a 28-Joint RA Disease Activity Score (DAS28-ESR), the doctor or patient Visual Analogue Scale (Dr. or PT-VAS), Steinbrocker's stage and class and the health assessment questionnaire disability index (HAQ). We also reviewed the information regarding current RA therapeutics including methotrexate, prednisolone, and biological agents. Other epidemiologic and anthropometric variables including age, duration of RA disease and body mass index (BMI) were extracted from the KURAMA database. Using a self-reported questionnaire form, we also collected information regarding history of falls and bone fractures in the previous year. The use of osteoporosis medication was obtained from the electronic medical record.

\section{Statistical analysis}

We present continuous variables as the mean (standard deviation (SD)) or as the median (interquartile range (IQR)) and categorial variables as numbers (\%). To compare baseline characteristics according to 25(OH)D status, we divided participants into the following two groups by the median of serum 25(OH)D concentration: low titer group (25(OH)D: $5.9-16.0 \mathrm{ng} / \mathrm{ml})$; high titer group (25(OH)D: $16.1-32.1 \mathrm{ng} / \mathrm{ml})$. We then performed a Mann-Whitney's U test or a Fisher's exact test for continuous variables and categorical variables, respectively. To explore the relationship between 25(OH)D status and severe sarcopenia, univariate and multivariate logistic regression analyses were conducted using serum 25(OH)D as a binary or continuous variable. In multivariate analyses, we constructed the following multiple models by incorporating significant variables in the univariate analysis and clinically relevant variables including RA therapeutics ${ }^{10,25}$ : model 1 was adjusted for $25(\mathrm{OH}) \mathrm{D}$ status, age, and body mass index; model 2 was adjusted for variables in model 1 plus nutrition status (MNA-SF) and RA-related factors (DAS28-ESR, Stage 3 and 4 vs. 1 and 2, HAQ, and therapeutics (use of prednisolone, biologics, and methotrexate)); model 3 was adjusted for variables in model 2 plus the prevalence of osteoporosis medication. We also adopted multivariate logistic regression analysis using the same models for each component of severe sarcopenia (low muscle mass, low muscle strength, and low physical performance). 
JMP 15.2.0 (SAS Institute Inc., Cary, NC, USA) was used for statistical analyses; a value of $\mathrm{P}<0.05$ was considered significant.

\section{Results}

Participant characteristics of this study are provided in Table 1. Overall, 156 older female RA patients with a mean (SD) age of 69.7 (6.7) were subjected to the following analysis. The median (IQR) serum 25(OH)D concentration was $16.0(12.8$ - 19.2), which represents a lower concentration than that in the general population ${ }^{26}$ and accords with that in other RA studies ${ }^{18}$. As for RA-related factors, the average (SD) duration of RA was 16.1 (12.7). Disease activity of RA by DAS28-ESR was generally under the following therapeutics: methotrexate in $67.3 \%$, prednisolone in $27.6 \%$, and biological agent in $35.3 \%$, but about twothirds of the patients had advanced joint deformity or destruction as assessed by a Steinbrocker's stage of $3(21.1 \%)$ or $4(46.8 \%)$.

Of these participants, on the basis of the diagnostic criteria of sarcopenia in AWGS $2019{ }^{23}, 46$ (29.5\%) were determined to have severe sarcopenia and 70 (44.9\%) were determined to have sarcopenia. Regarding the components of sarcopenia, mean (SD) of SMI, handgrip strength and gait speed were 5.64 (0.83), 14.5 (7.2) and $0.95(0.29)$, respectively. Under these conditions, $50.6 \%, 67.3 \%$, and $50.6 \%$ of the participants fulfilled the criterion for low muscle mass, low muscle strength (handgrip strength), or poor physical performance (gait speed), respectively.

\section{Comparison of clinical characteristics according to 25(OH)D status}

To determine whether serum 25(OH)D status was associated with RA-related factors and sarcopenia in the RA population, we first divided participants into two groups (low titer/high titer) at a cutoff point of the median serum 25(OH)D concentration and then compared their characteristics. As for the components of sarcopenia, skeletal muscle mass, handgrip strength and gait speed were significantly lower in the low 25(OH)D group (Table 2). The prevalence of severe sarcopenia was significantly higher in the low 25(OH)D group, which was also in the case in sarcopenia. Regarding RA-related factors, as in other reports ${ }^{27,28}$, DAS28-ESR, the degree of current disease activity, tended to be higher in the low $25(\mathrm{OH}) \mathrm{D}$ group. Regarding current RA therapeutics, the use rate of prednisolone was higher in the low $25(\mathrm{OH}) \mathrm{D}$ group; the use rate of methotrexate was lower in the low 25(OH)D group.

\section{Low 25(OH)D status is independently and positively associated with a high prevalence of severe sarcopenia}

Although it is emerging that low 25(OH)D concentrations affect the sarcopenia state in the general population, in a RA population these associations are not well understood. We therefore performed univariate and multivariate regression analyses with the presence of severe sarcopenia as a dependent variable. Univariate logistic regression analyses revealed that low 25(OH)D status was significantly associated with a high prevalence of severe sarcopenia (OR 4.25; 95\% Cl 1.99 - 9.09) (Table 3 left). Age, BMI, DAS28-ESR, Stage, HAQ score, the use of prednisolone and nutritional status (MNA-SF) 
were also associated with severe sarcopenia. We then conducted multivariate logistic regression analyses to clarify whether low 25(OH)D status independently contributes to severe sarcopenia. Among the factors that were significant in the univariate analysis, we excluded the HAQ score from covariates in the multivariate analyses because this score might be a result, not a cause, of sarcopenia. We then determined that low 25(OH)D status was independently associated with high prevalence of severe sarcopenia in model 1 adjusted for age, BMI, 25(OH)D status (OR 4.42; 95\% Cl 1.80 - 10.8) (Table 3 middle). In other models adjusted for RA-related and nutritional factors (model 2) or adjusted for factors that may affect $25(\mathrm{OH}) \mathrm{D}$ including osteoporosis and vitamin $\mathrm{D}$ medication (model 3 ), the same significant relationships still remained. When $25(\mathrm{OH}) \mathrm{D}$ was modeled as a continuous variable, lower 25(OH)D concentrations were also significantly associated with severe sarcopenia in all models (OR 0.91; 95\% Cl 0.84 - 0.99: model 1) (Supplementary Table S1).

\section{Low 25(OH)D status is independently associated with both low physical performance and low skeletal muscle mass}

We then conducted further multivariate analyses to clarify which components of severe sarcopenia were most associated with low serum 25(OH)D status. Regarding the components of severe sarcopenia, both low physical performance and low skeletal muscle mass were significantly associated with low serum 25(OH) status in model 1 (physical performance: OR 2.65; 95\% Cl $1.34-5.23$, skeletal muscle mass: OR 2.54; 95\% Cl 1.10 - 5.88) (Figure 1). The same associations were maintained in the other models (model 2 and 3). Other variables are described in supplementary Table S2. There was no significant association with muscle strength. Low $25(\mathrm{OH}) \mathrm{D}$ status is therefore independently associated with a high prevalence of severe sarcopenia and with both low physical performance and low skeletal muscle index mass among the components of sarcopenia.

\section{Discussion}

The present study is the first to document a significant association between vitamin D deficiency and severe sarcopenia in an RA population. Our participants had a high prevalence of severe sarcopenia (29.5\%) and vitamin D deficiency (median $16.0 \mathrm{ng} / \mathrm{ml}$ ); multivariate analyses showed low serum vitamin $D$ to be an independent risk for severe sarcopenia (OR 4.42). In addition, among the three components of sarcopenia, decreased gait speed and muscle mass showed significant associations with low serum vitamin $D$. These findings accord with those in general population studies: low serum vitamin $D$ has a stronger association with lower function on walking than on other physical performance or muscle strength ${ }^{29,30}$. Our results suggest that vitamin D deficiency may contribute to the development of severe sarcopenia and impaired lower limb performance and skeletal muscle in RA patients, as is the case in the general population (Fig. 2).

Recently, basic studies have provided insight into the mechanism of vitamin D action on skeletal muscle function, including modulation of muscle differentiation, oxidative stress, cellular metabolism and inflammatory condition ${ }^{13,31}$. The expression of vitamin $\mathrm{D}$ receptors on muscle cells decreases with aging 
and is implicated in the development of sarcopenia ${ }^{32}$. Vitamin D deficiency also contributes to skeletal muscle atrophy via dysregulation of oxidative stress, mitochondrial function and calcium homeostasis 33,34. Furthermore, vitamin $D$ is well known for its anti-inflammatory and immunomodulatory properties

35. It suppresses Th1 and Th17 cytokines (IL-2, IFN-y, TNF-a, IL-17, IL-21) as well as innate immune responses such as toll-like receptor signaling and antigen presentation ${ }^{36}$; it promotes the expression of Th2 cytokines (IL-4, IL-5, IL-9, IL-13) and the differentiation of regulatory T cells ${ }^{37,38}$. Given that Th1 and Th17 responses participate in the pathogenesis of RA ${ }^{39}$, vitamin D might well ameliorate sarcopenia in RA patients partly through attenuating chronic inflammation that results in muscle catabolism as well as through modulating muscle function via multiple physiological pathways.

Similarly, epidemiological studies have shown clinical correlations between vitamin D deficiency and sarcopenia. In general populations, low serum vitamin D levels significantly associate with increased prevalence of sarcopenia and loss of physical performance such as walking speed ${ }^{14,15}$. Several metaanalyses and RCTs have also shown the positive effects of vitamin D supplementation, especially on lower limb muscles ${ }^{16,17}$. In RA populations, however, the relationship between sarcopenia and vitamin D status has not been clarified even though the prevalence of sarcopenia and vitamin D insufficiency is markedly higher in the RA population than it is in the general population ${ }^{6,40}$. The present study provides novel evidence that low serum vitamin $D$ is a significant risk factor of severe sarcopenia in RA. Taking these findings together, vitamin $\mathrm{D}$ deficiency may well be a candidate co-adjuvant therapeutic target to prevent severe sarcopenia in RA patients; prospective studies of vitamin D supplementation to prevent sarcopenia in RA will be worthwhile.

There are several limitations in the present study. The present cross-sectional study does not imply causation and further prospective investigation is needed. There is also the possibility of selection bias because the study included only females and patients older than 60 years, although these are the dominant populations in RA. In addition, there might remain unadjusted confounding variables related to vitamin D status and sarcopenia including dietary profile, seasonal variation and socioeconomic status. Some information including history of falls and bone fractures was assessed by the recall method.

In conclusion, vitamin D deficiency is significantly associated with severe sarcopenia, low physical performance and low skeletal muscle mass index in an RA population. The therapeutic potential of vitamin D supplementation for sarcopenia should be investigated further in RA patients.

\section{Declarations}

\section{Acknowledgments}

We thank Ms. N. Kitayama and Ms. M. Yoneyama for the support of the patients. We also thank S. Nakagawa and M. lida for their technical assistance.

\section{Funding}


This study (KURAMA cohort study) is supported by AMED (the Japan Agency for Medical Research and Development) under Grant Number JP20ek0410069, JSPS KAKENHI Grant Number JP 19K11165 and a grant from Daiichi Sankyo Co. Ltd. The funder had no role in the design of the study, the collection or analysis of the data, the writing of the manuscript, or the decision to submit the manuscript for publication.

\section{Competing interests}

K.M. (Murata), and M.T. (Tanaka) are members of a department financially supported by Nagahama City, Shiga, Japan, Toyooka City, Hyogo, Japan and five pharmaceutical companies (Tanabe-Mitsubishi, Chugai, UCB Japan, Ayumi and Asahi-Kasei). R.W. receives speaker's fee from Mitsubishi Tanabe Pharma, Pfizer, Sanofi, AbbVie, Asahi Kasei, Eisai, Eli Lilly, Bristol-Myers Squibb, and Janssen. H.I. receives a research grant and/or speaker fee from Bristol-Myers, Kyocera, and Asahi-Kasei. M.T. (Tanaka) has received research grants and/or speaker fees from AbbVie GK, Asahi Kasei Pharma Corp., Astellas Pharma Inc., Ayumi Pharmaceutical Corp., Bristol-Myers Squibb, Chugai Pharmaceutical Co., Ltd., Eisai Co., Ltd., Eli Lilly Japan K.K., Pfizer Inc., UCB Japan Co., Ltd., Janssen Pharmaceutical K.K., Mitsubishi Tanabe Pharma Corp., Novartis Pharma K.K., Taisho Pharma Co., Ltd. S.M. receives grants and/or speaker fees from Daiichi Sankyo, Asahi-Kasei, Chugai, Ayumi, and Tanabe Mitsubishi. A.M. receives speaking fees and/or research grants from Eli Lilly Japan K.K., Ono Pharmaceutical Co., Pfizer Inc., UCB Japan, AbbVie G.K., Asahi Kasei Pharma and Chugai Pharmaceutical Co. Ltd. M.H. receives grants and /or speaker fees from Bristol-Meyers, Eisa, Eli Lilly, Novartis Pharma, and Tanabe Mitsubishi. H.M., M.K., M.T.(Torii), W.Y., Y.F., K.I., E.O., K.M.(Murakami), K.M. (Murata), H.A., and N.I. declare no conflicts of interest. The sponsors had no role in the design of the study, the collection or analysis of the data, the writing of the manuscript, or the decision to submit the manuscript for publication. The authors, their immediate families, and any research foundations with which they are affiliated have not received any financial payments or other benefits from any commercial entity related to the subject of this article.

\section{Author Contributions}

H.M. and M.T. (Torii) are responsible for study conception and design. H.M. and M.K contributed to the interpretation of the data, drafted the manuscript, and revised the manuscript. M.H. contributed to interpretation of the data and revised the manuscript. W.Y., Y.F., K.I., E.O., K.M.(Murakami), R.W., K.M. (Murata), H.I., M.T.(Tanaka), H.A., S.M., A.M., and N.I. contributed to supervision of the manuscript for intellectual content. All authors have approved the final manuscript for publication and have agreed to be personally accountable for the authors' contributions.

\section{References}

1. Chen, L. K. et al. Sarcopenia in Asia: consensus report of the Asian Working Group for Sarcopenia. $J$ Am Med Dir Assoc, 15, 95-101 https://doi.org/10.1016/j.jamda.2013.11.025 (2014). 
2. Beaudart, C., Zaaria, M., Pasleau, F., Reginster, J. Y. \& Bruyere, O. Health Outcomes of Sarcopenia: A Systematic Review and Meta-Analysis. PLoS One, 12, e0169548 https://doi.org/10.1371/journal.pone.0169548 (2017).

3. Yeung, S. S. Y. et al. Sarcopenia and its association with falls and fractures in older adults: A systematic review and meta-analysis. J Cachexia Sarcopenia Muscle, 10, 485-500 https://doi.org/10.1002/jcsm.12411 (2019).

4. Bowen, T. S., Schuler, G. \& Adams, V. Skeletal muscle wasting in cachexia and sarcopenia: molecular pathophysiology and impact of exercise training. J Cachexia Sarcopenia Muscle, 6, 197-207 https://doi.org/10.1002/jcsm.12043 (2015).

5. Yoshimura, Y. et al. Interventions for Treating Sarcopenia: A Systematic Review and Meta-Analysis of Randomized Controlled Studies. J Am Med Dir Assoc 18, 553 e551-553 e516, doi:10.1016/j.jamda.2017.03.019 (2017).

6. Petermann-Rocha, F. et al. Factors associated with sarcopenia: A cross-sectional analysis using UK Biobank. Maturitas, 133, 60-67 https://doi.org/10.1016/j.maturitas.2020.01.004 (2020).

7. Sokka, T., Hakkinen, A., Krishnan, E. \& Hannonen, P. Similar prediction of mortality by the health assessment questionnaire in patients with rheumatoid arthritis and the general population. Ann Rheum Dis, 63, 494-497 https://doi.org/10.1136/ard.2003.009530 (2004).

8. Cramer, J. T. et al. Impacts of High-Protein Oral Nutritional Supplements Among Malnourished Men and Women with Sarcopenia: A Multicenter, Randomized, Double-Blinded, Controlled Trial. J Am Med Dir Assoc, 17, 1044-1055 https://doi.org/10.1016/j.jamda.2016.08.009 (2016).

9. Cruz-Jentoft, A. J., Sayer, A. A. \& Sarcopenia The Lancet, 393, 2636-2646 https://doi.org/10.1016/s0140-6736(19)31138-9 (2019).

10. Torii, M. et al. Prevalence and factors associated with sarcopenia in patients with rheumatoid arthritis. Mod Rheumatol, 29, 589-595 https://doi.org/10.1080/14397595.2018.1510565 (2019).

11. Wu, C. H. et al. Prevalence and associated factors of sarcopenia and severe sarcopenia in older Taiwanese living in rural community: the Tianliao Old People study 04. Geriatr Gerontol Int, 14 (Suppl 1), 69-75 https://doi.org/10.1111/ggi.12233 (2014).

12. Giles, J. T. et al. Abnormal body composition phenotypes in older rheumatoid arthritis patients: association with disease characteristics and pharmacotherapies. Arthritis Rheum, 59, 807-815 https://doi.org/10.1002/art.23719 (2008).

13. Dzik, K. P. \& Kaczor, J. J. Mechanisms of vitamin D on skeletal muscle function: oxidative stress, energy metabolism and anabolic state. Eur J Appl Physiol, 119, 825-839 https://doi.org/10.1007/s00421-019-04104-x (2019).

14. Wang, J. et al. Vitamin D is related to handgrip strength in adult men aged 50 years and over: A population study from the TCLSIH cohort study. Clin Endocrinol (Oxf), 90, 753-765 https://doi.org/10.1111/cen.13952 (2019).

15. Wicherts, I. S. et al. Vitamin D status predicts physical performance and its decline in older persons. $J$ Clin Endocrinol Metab, 92, 2058-2065 https://doi.org/10.1210/jc.2006-1525 (2007). 
16. Beaudart, C. et al. The effects of vitamin D on skeletal muscle strength, muscle mass, and muscle power: a systematic review and meta-analysis of randomized controlled trials. J Clin Endocrinol Metab, 99, 4336-4345 https://doi.org/10.1210/jc.2014-1742 (2014).

17. Gkekas, N. K. et al. The effect of vitamin D plus protein supplementation on sarcopenia: A systematic review and meta-analysis of randomized controlled trials. Maturitas, 145, 56-63 https://doi.org/10.1016/j.maturitas.2021.01.002 (2021).

18. Furuya, T. et al. Prevalence of and factors associated with vitamin D deficiency in 4,793 Japanese patients with rheumatoid arthritis. Clin Rheumatol, 32, 1081-1087 https://doi.org/10.1007/s10067013-2216-4 (2013).

19. Hashimoto, M. et al. Increase of hemoglobin levels by anti-IL-6 receptor antibody (tocilizumab) in rheumatoid arthritis. PLoS One, 9, e98202 https://doi.org/10.1371/journal.pone.0098202 (2014).

20. Minamino, H. et al. Urinary sodium-to-potassium ratio associates with hypertension and current disease activity in patients with rheumatoid arthritis: a cross-sectional study. Arthritis Res Ther, 23, 96 https://doi.org/10.1186/s13075-021-02479-x (2021).

21. Singh, J. A. et al. 2015 American College of Rheumatology Guideline for the Treatment of Rheumatoid Arthritis. Arthritis Care Res (Hoboken) 68, 1-25, doi:10.1002/acr.22783 (2016).

22. Minamino, H. et al. Habitual fish intake negatively correlates with prevalence of frailty among patients with rheumatoid arthritis. Sci Rep, 11, 5104 https://doi.org/10.1038/s41598-021-84479-0 (2021).

23. Chen, L. K. et al. Asian Working Group for Sarcopenia: 2019 Consensus Update on Sarcopenia Diagnosis and Treatment. J Am Med Dir Assoc 21, 300-307 e302, doi:10.1016/j.jamda.2019.12.012 (2020).

24. Rubenstein, L. Z., Harker, J. O., Salva, A., Guigoz, Y. \& Vellas, B. Screening for undernutrition in geriatric practice: developing the short-form mini-nutritional assessment (MNA-SF). J Gerontol A Biol Sci Med Sci, 56, M366-372 https://doi.org/10.1093/gerona/56.6.m366 (2001).

25. Yamada, Y. et al. Glucocorticoid use is an independent risk factor for developing sarcopenia in patients with rheumatoid arthritis: from the CHIKARA study. Clin Rheumatol, 39, 1757-1764 https://doi.org/10.1007/s10067-020-04929-4 (2020).

26. Wong, Y. Y., McCaul, K. A., Yeap, B. B., Hankey, G. J. \& Flicker, L. Low vitamin D status is an independent predictor of increased frailty and all-cause mortality in older men: the Health in Men Study. J Clin Endocrinol Metab, 98, 3821-3828 https://doi.org/10.1210/jc.2013-1702 (2013).

27. Haque, U. J. \& Bartlett, S. J. Relationships among vitamin D, disease activity, pain and disability in rheumatoid arthritis. Clinical and Experimental Rheumatology, 28, 745-747 (2010).

28. Rossini, M. et al. Vitamin D deficiency in rheumatoid arthritis: prevalence, determinants and associations with disease activity and disability. Arthritis Res Ther, 12, R216 https://doi.org/10.1186/ar3195 (2010).

29. Toffanello, E. D. et al. Vitamin D and physical performance in elderly subjects: the Pro.V.A study. PLoS One, 7, e34950 https://doi.org/10.1371/journal.pone.0034950 (2012). 
30. Vaes, A. M. M. et al. The association between 25-hydroxyvitamin D concentration, physical performance and frailty status in older adults. Eur J Nutr, 58, 1173-1181 https://doi.org/10.1007/s00394-018-1634-0 (2019).

31. Jain, S. K., Parsanathan, R., Achari, A. E., Kanikarla-Marie, P. \& Bocchini, J. A. Jr. Glutathione Stimulates Vitamin D Regulatory and Glucose-Metabolism Genes, Lowers Oxidative Stress and Inflammation, and Increases 25-Hydroxy-Vitamin D Levels in Blood: A Novel Approach to Treat 25Hydroxyvitamin D Deficiency. Antioxid Redox Signal, 29, 1792-1807 https://doi.org/10.1089/ars.2017.7462 (2018).

32. Bischoff-Ferrari, H. A. et al. Vitamin D receptor expression in human muscle tissue decreases with age. J Bone Miner Res, 19, 265-269 https://doi.org/10.1359/jbmr.2004.19.2.265 (2004).

33. Ryan, Z. C. et al. 1 alpha,25-Dihydroxyvitamin D3 Regulates Mitochondrial Oxygen Consumption and Dynamics in Human Skeletal Muscle Cells. J Biol Chem, 291, 1514-1528 https://doi.org/10.1074/jbc.M115.684399 (2016).

34. Sinha, A., Hollingsworth, K. G., Ball, S. \& Cheetham, T. Improving the vitamin D status of vitamin D deficient adults is associated with improved mitochondrial oxidative function in skeletal muscle. $J$ Clin Endocrinol Metab, 98, E509-513 https://doi.org/10.1210/jc.2012-3592 (2013).

35. Charoenngam, N. \& Holick, M. F. Immunologic Effects of Vitamin D on Human Health and Disease. Nutrients 12, doi:10.3390/nu12072097 (2020).

36. Tang, J. et al. Calcitriol suppresses antiretinal autoimmunity through inhibitory effects on the Th17 effector response. J Immunol, 182, 4624-4632 https://doi.org/10.4049/jimmunol.0801543 (2009).

37. Boonstra, A. et al. 1alpha,25-Dihydroxyvitamin d3 has a direct effect on naive CD4(+) T cells to enhance the development of Th2 cells. J Immunol, 167, 4974-4980 https://doi.org/10.4049/jimmunol.167.9.4974 (2001).

38. Gregori, S., Giarratana, N., Smiroldo, S., Uskokovic, M. \& Adorini, L. A 1alpha,25-dihydroxyvitamin D(3) analog enhances regulatory T-cells and arrests autoimmune diabetes in NOD mice., 51, 1367-1374 https://doi.org/10.2337/diabetes.51.5.1367 (2002).

39. McInnes, I. B. \& Schett, G. Cytokines in the pathogenesis of rheumatoid arthritis. Nat Rev Immunol, 7, 429-442 https://doi.org/10.1038/nri2094 (2007).

40. Mouterde, G. et al. Association Between Vitamin D Deficiency and Disease Activity, Disability, and Radiographic Progression in Early Rheumatoid Arthritis: The ESPOIR Cohort. J Rheumatol, 47, 16241628 https://doi.org/10.3899/jrheum.190795 (2020).

\section{Tables}

\section{Table 1. Characteristics of this study population}

Data are presented as the mean (standard deviation (SD)) or as the median (interquartile range (IQR)) for continuous variables, and as numbers (\%) for categorial variables. 


\begin{tabular}{|c|c|c|}
\hline & RA patients & * Steinbrocker's \\
\hline Characteristics & $(N=156)$ & class \\
\hline Age, mean (SD), years & $69.7(6.7)$ & Abbreviations: $R A$ \\
\hline Body composition and physical activity parameters & & $\begin{array}{l}\text { rheumatoid arthritis, MNA- } \\
\text { SF Mini Nutritional }\end{array}$ \\
\hline Body mass index, mean (SD), $\mathrm{kg} / \mathrm{m}^{2}$ & $22.0(3.6)$ & Assessment Short-Form, \\
\hline Skeletal mass index, mean (SD), $\mathrm{kg} / \mathrm{m}^{2}$ & $5.64(0.83)$ & $\begin{array}{l}D A S 28 \text { disease activity score } \\
\text { using } 28 \text { joints, VAS visual }\end{array}$ \\
\hline Handgrip strength - dominant, mean (SD), kg & $14.5(7.2)$ & analogue scale, $H A Q$ health \\
\hline Gait speed, mean (SD), m/s & $0.95(0.29)$ & assessment questionnaire, \\
\hline Sarcopenia (+), $n(\%)$ & $70(44.9)$ & Table 2. Characteristics of \\
\hline Severe sarcopenia $(+), n(\%)$ & $46(29.5)$ & $\begin{array}{l}\text { particlpants by serum } \\
25(\mathrm{OH}) \mathrm{D} \text { status }\end{array}$ \\
\hline Any fall in the previous year, $n(\%)$ & $25(16.2)$ & \\
\hline Any fracture in the previous year, $n(\%)$ & $7(4.6)$ & RA patients are divided into \\
\hline Osteoporosis medication, $n(\%)$ & $45(28.9)$ & the following two groups by \\
\hline MNA-SF, mean (SD) & $12.0(2.0)$ & $\begin{array}{l}\text { median of serum } 25(\mathrm{OH}) \mathrm{D} \text { : } \\
\text { low titer group }(25(\mathrm{OH}) \mathrm{D}: 5.9\end{array}$ \\
\hline RA disease characteristics & & $-16.0 \mathrm{ng} / \mathrm{ml})$ and high titer \\
\hline Duration, mean (SD), years & $16.1(12.7)$ & group (25(OH)D: 16.1 - 32.1 \\
\hline DAS28-ESR, mean (SD) & $2.96(0.98)$ & $\begin{array}{l}\mathrm{ng} / \mathrm{ml}) \text {. Data are presented } \\
\text { as the mean }( \pm \text { standard }\end{array}$ \\
\hline HAQ score, mean (SD) & $0.83(0.74)$ & deviation) or as the median \\
\hline Stage*, mean $(S D)^{*}$ & $3.01(1.10)$ & (interquartile range (IQR)) for \\
\hline Stage $1, n(\%)$ & $21(13.4)$ & numbers (\%) for categorial \\
\hline Stage $2, n(\%)$ & $29(18.6)$ & variables. \\
\hline Stage $3, n(\%)$ & $33(21.1)$ & Abbreviations: $R A$ \\
\hline Stage $4, n(\%)$ & $73(46.8)$ & rheumatoid arthritis, $M N A$ - \\
\hline Class*, mean (SD) & $1.82(0.60)$ & $\begin{array}{l}\text { SF Mini Nutritional } \\
\text { Assessment Short-Form, }\end{array}$ \\
\hline Current RA medications & & DAS28 disease activity score \\
\hline Methotrexate use, $n(\%)$ & $105(67.3)$ & using 28 joints, VAS visual \\
\hline Prednisolone use, $n(\%)$ & $43(27.6)$ & assessment questionnaire, \\
\hline Biological agent use, $n(\%)$ & $55(35.3)$ & Table 3. Logistic analyses \\
\hline Laboratory data & & for independent factors \\
\hline
\end{tabular}


Serum 25(OH) D, median (IQR), ng/ml

CRP, median (IQR), mg/dL
$16.0(12.8-19.2)$

$0.1(0.075-0.30)$ associated with severe

sarcopenia.

Results of univariate (left) and multivariate (right) logistic analyses for independent variables associated with severe sarcopenia. Model 1 was adjusted for vitamin D status, age, and body mass index. Model 2 was adjusted for variables in model 1 plus nutrition status (MNA-SF), and RA-related factors (DAS28-ESR, Stage, HAQ, and therapeutics (use of prednisolone, biologics, and methotrexate)). Model 3 was adjusted for variables in model 2 plus the prevalence of osteoporosis medication.

Abbreviations: RA rheumatoid arthritis, DAS28 disease activity score using 28 joints, $H A Q$ health assessment questionnaire, MNA-SF Mini Nutritional Assessment Short-Form

\section{Figures}

\begin{tabular}{|c|c|c|c|c|}
\hline Dependent variable & & Odds ratio $(95 \% \mathrm{Cl})$ & & $P$ value \\
\hline \multirow[t]{3}{*}{ Low physical performance } & Model 1 & $\longmapsto-1$ & $2.65(1.34-5.23)$ & 0.0043 \\
\hline & Model 2 & $\bullet-1$ & $2.35(1.11-4.98)$ & 0.024 \\
\hline & Model 3 & $\longrightarrow$ & $2.37(1.11-5.04)$ & 0.024 \\
\hline \multirow[t]{3}{*}{ Low muscle strength } & Model 1 & $\vdots$ & $1.22(0.41-1.64)$ & 0.58 \\
\hline & Model 2 & 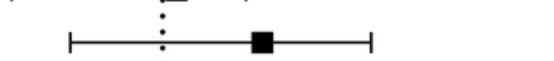 & $1.41(0.58-3.44)$ & 0.45 \\
\hline & Model 3 & $\vdots$ & $1.42(0.57-3.56)$ & 0.45 \\
\hline \multirow[t]{3}{*}{ Low muscle mass } & Model 1 & $\vdots$ & $2.54(1.10-5.88)$ & 0.027 \\
\hline & Model 2 & $\longmapsto 0$ & $3.38(1.30-8.77)$ & 0.010 \\
\hline & Model 3 & 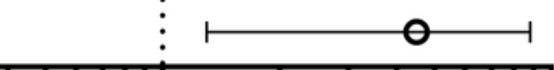 & $3.39(1.30-8.82)$ & 0.010 \\
\hline
\end{tabular}

\section{Figure 1}

Associations between vitamin D status and components of sarcopenia Results of multivariate logistic analyses for independent variables associated with components of sarcopenia. This forest plot represents the odds ratio and $95 \%$ confidence interval $(\mathrm{Cl})$ for each sarcopenia-related component in each adjusted model. Model 1 was adjusted for vitamin D status, age, and body mass index. Model 2 was adjusted for variables in model 1 plus nutrition status (MNA-SF) and RA-related factors (DAS28-ESR, 


\section{Low titer $(n=78) \quad$ High titer $(n=78)$}

\begin{tabular}{llll}
\hline 25(OH)D level (range, $\mathrm{ng} / \mathrm{ml})$ & $5.9-16.0$ & $16.1-32.1$ & $P$ value \\
\hline Age, mean (SD), year & $70.4(6.9)$ & $69.1(6.4)$ & 0.21 \\
\hline Body mass index, mean (SD), kg/m² & $21.7(3.5)$ & $22.2(3.6)$ & 0.29 \\
\hline Factors associated with sarcopenia & & & \\
\hline Skeletal mass index, mean (SD), kg/m² & $5.45(0.90)$ & $5.83(0.69)$ & $\mathbf{0 . 0 0 3 6}$ \\
\hline Handgrip strength - dominant, mean (SD), kg & $13.1(7.6)$ & $16.0(6.5)$ & $\mathbf{0 . 0 0 9 4}$ \\
\hline Gait speed, mean (SD), m/s & $0.88(0.30)$ & $1.02(0.27)$ & $\mathbf{0 . 0 0 2 5}$ \\
\hline Sarcopenia (+), $n(\%)$ & $44(56.4)$ & $26(33.3)$ & $\mathbf{0 . 0 0 3 6}$ \\
\hline Severe sarcopenia (+), $n(\%)$ & $34(43.6)$ & $12(15.4)$ & $<0.0001$ \\
\hline Osteoporosis medication, $n(\%)$ & $41(35.0)$ & $36(29.8)$ & 1.00 \\
\hline MNA-SF, mean (SD) & $11.9(2.1)$ & $12.0(1.9)$ & 0.69
\end{tabular}

RA disease characteristics

\begin{tabular}{llll} 
Disease duration, mean (SD), year & $16.6(13.6)$ & $15.6(11.7)$ & 0.63 \\
\hline DAS28-ESR, mean (SD) & $3.11(1.04)$ & $2.81(0.90)$ & 0.055 \\
\hline CRP, median (IQR), mg/dL & $0.1(0-0.4)$ & $0.1(0.1-0.3)$ & 0.44 \\
\hline HAQ, mean (SD) & $1.00(0.79)$ & $0.67(0.66)$ & 0.0096 \\
\hline Stage, mean (SD) & $2.99(1.10)$ & $3.04(1.10)$ & 0.77 \\
\hline Stage 4, $n(\%)$ & $35(44.9)$ & $38(48.7)$ & 0.63 \\
Stage 3 and 4, $n(\%)$ & $53(68.0)$ & $53(68.0)$ & 1.00 \\
Stage 2, 3 and 4, $n(\%)$ & $67(85.9)$ & $68(87.2)$ & 0.81 \\
\hline Class, mean (SD) & $1.91(0.65)$ & $1.73(0.53)$ & 0.060
\end{tabular}

Current therapeutic agent

Methotrexate use, $n(\%)$

\begin{tabular}{lll}
$49(62.8)$ & $56(71.8)$ & 0.23 \\
$29(37.2)$ & $26(33.3)$ & 0.61 \\
\hline $28(35.9)$ & $15(19.2)$ & 0.019
\end{tabular}

Stage, HAQ, and therapeutics (use of prednisolone, biologics, and methotrexate)). Model 3 was adjusted for variables in model 2 plus the prevalence of osteoporosis medication. 


\begin{tabular}{|c|c|c|c|c|c|c|c|c|}
\hline & & & model 1 & & model 2 & & model 3 & \\
\hline variables & $\begin{array}{l}\text { OR } \\
(95 \% \\
\text { Cl) }\end{array}$ & $P$ value & $\begin{array}{l}\text { OR } \\
(95 \% \\
\text { Cl) }\end{array}$ & $P$ value & $\begin{array}{l}\text { OR } \\
\text { (95\% } \\
\text { Cl) }\end{array}$ & $P$ value & $\begin{array}{l}\text { OR } \\
\text { (95\% } \\
\text { Cl) }\end{array}$ & $P$ value \\
\hline Age (1 year) & $\begin{array}{l}1.15 \\
(1.08- \\
1.22)\end{array}$ & $\begin{array}{l}<.0001 \\
0.00\end{array}$ & $\begin{array}{l}1.17 \\
(1.09- \\
1.25)\end{array}$ & $\begin{array}{l}<.0001 \\
0.0\end{array}$ & $\begin{array}{l}1.21 \\
(1.10- \\
1.33)\end{array}$ & $\begin{array}{l}<.0001 \\
\end{array}$ & $\begin{array}{l}1.21 \\
(1.10- \\
1.33)\end{array}$ & $\begin{array}{l}<.0001 \\
0.00\end{array}$ \\
\hline $\begin{array}{l}\text { Body mass } \\
\text { index }\left(1 \mathrm{~kg} / \mathrm{m}^{2}\right)\end{array}$ & $\begin{array}{l}0.76 \\
(0.67- \\
0.86)\end{array}$ & $\begin{array}{l}< \\
0.0001\end{array}$ & $\begin{array}{l}0.72 \\
(0.62- \\
0.84)\end{array}$ & $\begin{array}{l}<.0001 \\
0.0\end{array}$ & $\begin{array}{l}0.76 \\
(0.60- \\
0.95)\end{array}$ & 0.0085 & $\begin{array}{l}0.76 \\
(0.60- \\
0.95)\end{array}$ & 0.010 \\
\hline 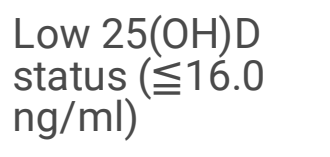 & $\begin{array}{l}4.25 \\
(1.99- \\
9.09)\end{array}$ & $\begin{array}{l}< \\
0.0001\end{array}$ & $\begin{array}{l}4.42 \\
(1.80- \\
10.8)\end{array}$ & 0.0007 & $\begin{array}{l}5.92 \\
(1.98- \\
17.7)\end{array}$ & 0.0006 & $\begin{array}{l}6.00 \\
(1.99- \\
18.08)\end{array}$ & 0.0006 \\
\hline DAS28-ESR & $\begin{array}{l}1.58 \\
(1.09- \\
2.31)\end{array}$ & 0.015 & & & $\begin{array}{l}1.03 \\
(0.62- \\
1.74)\end{array}$ & 0.90 & $\begin{array}{l}1.23 \\
(0.86- \\
1.76)\end{array}$ & 0.88 \\
\hline $\begin{array}{l}\text { Stage (3, } 4 \text { vs. } 1 \text {, } \\
\text { 2) }\end{array}$ & $\begin{array}{l}4.44 \\
(1.74- \\
11.4)\end{array}$ & 0.0005 & & & $\begin{array}{l}4.33 \\
(1.33- \\
14.08)\end{array}$ & 0.0010 & $\begin{array}{l}4.40 \\
(1.35- \\
14.32)\end{array}$ & 0.0097 \\
\hline HAQ & $\begin{array}{l}4.03 \\
(2.19- \\
7.41)\end{array}$ & $\begin{array}{l}< \\
0.0001\end{array}$ & & & & & & \\
\hline $\begin{array}{l}\text { Methotrexate } \\
\text { use }\end{array}$ & $\begin{array}{l}0.58 \\
(0.28- \\
1.19)\end{array}$ & 0.14 & & & $\begin{array}{l}2.18 \\
(0.66- \\
7.22)\end{array}$ & 0.19 & $\begin{array}{l}2.18 \\
(0.66- \\
7.22)\end{array}$ & 0.19 \\
\hline $\begin{array}{l}\text { Prednisolone } \\
\text { use }\end{array}$ & $\begin{array}{l}2.91 \\
(1.39- \\
6.11)\end{array}$ & 0.0049 & & & $\begin{array}{l}2.45 \\
(0.74- \\
8.06)\end{array}$ & 0.09 & $\begin{array}{l}2.45 \\
(0.74- \\
8.06)\end{array}$ & 0.13 \\
\hline $\begin{array}{l}\text { Biological } \\
\text { agents use }\end{array}$ & $\begin{array}{l}0.47 \\
(0.21- \\
1.03)\end{array}$ & 0.058 & & & $\begin{array}{l}0.70 \\
(0.22- \\
2.21)\end{array}$ & 0.52 & $\begin{array}{l}0.70 \\
(0.22- \\
2.21)\end{array}$ & 0.54 \\
\hline MNA-SF & $\begin{array}{l}0.70 \\
(0.59- \\
0.85)\end{array}$ & $\begin{array}{l}< \\
0.0001\end{array}$ & & & $\begin{array}{l}0.91 \\
(0.66- \\
1.24)\end{array}$ & 0.54 & $\begin{array}{l}0.90 \\
(0.66- \\
1.24)\end{array}$ & 0.53 \\
\hline $\begin{array}{l}\text { Osteoporosis } \\
\text { medication (+) }\end{array}$ & $\begin{array}{l}1.72 \\
(0.82- \\
3.59)\end{array}$ & 0.15 & & & & & $\begin{array}{l}1.22 \\
(0.40- \\
3.72)\end{array}$ & 0.73 \\
\hline
\end{tabular}




\section{In RA population....}

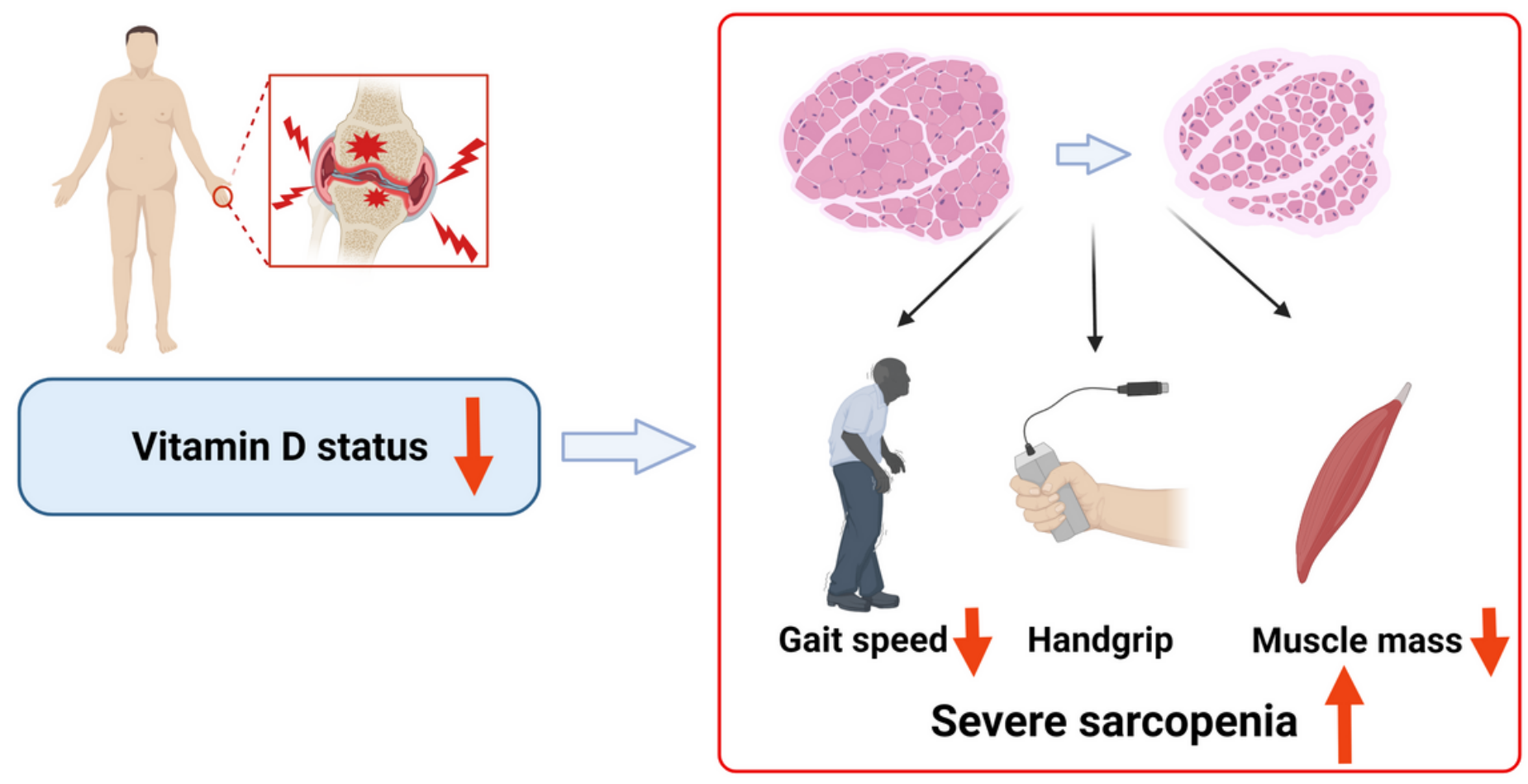

Figure 2

A proposed model of the relationship between vitamin D status, severe sarcopenia and its components in RA patients In this study, vitamin D deficiency were strongly associated with to increased prevalence of severe sarcopenia and impaired lower limb performance and skeletal muscle in RA patients. Modification strategy of vitamin D status including vitamin $D$ supplementation may contribute to the improvement of sarcopenia in RA. Abbreviations: RA rheumatoid arthritis

\section{Supplementary Files}

This is a list of supplementary files associated with this preprint. Click to download.

- SupplementaryTable1and2.docx 